\title{
Imaging Region of Interest
}

National Cancer Institute

\section{Source}

National Cancer Institute. Imaging Region of Interest. NCI Thesaurus. Code C85402.

A specific area of interest defined by a sequence of image overlays or a sequence of contours described as a single point (for a point ROI) or more than one point (representing and open or closed polygon). 